NASA/ASEE Summer Faculty Fellowship Program

Marshall Space Flight Center

The University of Alabama Huntsville

Characturization of Thunderstorm Induced Maxwell CuRRent Densties in the Middle AtMosphere

PREPARED BY:

ACADEMIC RANK:

UNIVERSITY AND

DEPARTMENT:

NASA/MSFC:

LABORATORY:

DIVISION:

BRANCH:

vigro colleague:

DATE:

CONIRACT NO:
Michael Edward BagINSKI

ASSISTANT PROFESSOR

AUBURN UNIVERSITY

DEPARTMENT OF ELECTRICAL ENGINEERING

SPACE SCIENCE

EARTH SCIENCE and Application

REMOTE SENSING

Cr. HUGH Christian

Dr. Richard BlakesLeE

SEPTEMBER 1, 1989

NGT-01-008-021

THE UnIVERSITY OF Alabama HuntSVILle 



\title{
Characterization of Thunderstorm InduCEd Maxwell CuRRENT Densities in the MidDle ATMOSPhere
}

by

\author{
Michael Edward Baginski \\ Assistant Professor of Electrical Engineering \\ Auburn University \\ Auburn, Alabama
}

\begin{abstract}
Middle atmospheric transient Maxwell current densities generated by lightning induced charge perturbations are investigated via a simulation of Maxwell's equations. A time domain finite element analysis is employed for the simulations. The atmosphere is modeled as a region contained within a right circular cylinder with a height of $110 \mathrm{~km}$ and radius of $80 \mathrm{~km}$. A composite conductivity profile based on measured data is used with charge perturbations are centered about the vertical axis at altitucies of 6 and $10 \mathrm{~km}$. The simulations indicate that the temporal structure of the Maxwell current density is relatively insensitive to altitude variation within the region considered. It is also shown that the electric field and Maxwell current density are not generally aligned.
\end{abstract}




\section{ACKNOWLEDGEMENTS}

I would like to thank NASA/ASEE for allowing me to participate in the Summer Faculty Program at Marshall Space Flight Center. The Program was well coordinated by Mr. Frank Six of NASA and Dr. Gerald F. Karr of UAH. Dr. Hugh Christian, Dr. Richard Blakeslee and Dr. James Arnold were especially helpful in obtaining the necessary computer software and technical materials required for the research.

This summer's research endeavors have lead to the submittal of a manuscript to The Journal of Geophysical Research. This would not have been possible without the assistance of Dr. Hugh Christian and Dr. Richard Blakeslee. My appreciation for the personal interest both of these scientists have taken in the research can not be stated strongly enough. I am looking forward to future research in the area of Atmospheric Electricity with Dr. Christian and Dr. Blakeslee 


\section{INTRODUCTION}

The use of Maxwell currents and current densities to descrioe the electromagnetic response of the atmosphere is a fairly recent development. Krider and Musser and Nisbet have suggested that the thunderstorm is fundamentally a current source and should therefore be investigated in terms of current densities rather than electric fields. The current densities generated by charge perturbations associated with lightning consist of either conduction (resulting from charge movement) or displacement $(\varepsilon \partial E / \partial t)$ terms. The sum of these current densities are referred to as the Maxwell current density $\left(\mathrm{J}_{\mathrm{m}}\right)$.

$$
\begin{aligned}
& \mathrm{J}_{\mathrm{m}}=\nabla \times \mathrm{H}=\mathrm{J}_{\mathrm{p}}+\varepsilon \partial \mathrm{E} / \partial \mathrm{t} \\
& \mathrm{J}_{\mathrm{m}}=\text { Maxwell current density } \\
& \mathrm{J}_{\mathrm{p}}=\text { the sum of all conduction current } \\
& \text { densities including source terms } \\
& \varepsilon \partial \mathrm{E} / \partial \mathrm{t}=\text { displacement current density }
\end{aligned}
$$

The Maxwell current density has several properties that may be axploited to more accurately describe both the local and global effects of lightning on the atmosphere. Probably the most important of these is that the divergence of the Maxwell current density is zero $\left(\nabla \cdot \mathrm{J}_{\mathrm{m}}=0\right)$ making it a solenoidal quantity. As a consequence of this, the lines of the Maxwell current density form closed loops. Along these Maxwell curment density stramines the displacement term is usually dominant at low altitudes with the conduction current density dominating the high altitude Maxwell current density.

This solenoidal character of the Maxwell current density implies ihat the electrical parameters of the entire path of circulation stiongly affect its response and conversely; if the Maxwell current dentiat, along the streamline is mathematically describable, the com wonding streamline's electric field may be formulated by a simple 
time domain integration. An analogy exemplifying this phenomenon can be made to a source driving a series ladder network with each eiement consisting of a parallel $\mathrm{RC}$. The relative analogies in the circuit are as follows: $\mathrm{J}_{\mathrm{m}} \sim \mathrm{I}_{\mathrm{s}}$, voltage $\sim \mathrm{E}, \mathrm{C} \partial \mathrm{V} / \partial \mathrm{t} \sim \varepsilon \partial \mathrm{E} / \partial \mathrm{t}, \mathrm{V} / \mathrm{R} \sim \sigma \mathrm{E}$. It is obvious that the total impedance of the circuit limits the current response, analogous to the peak surge impedance along the streamline's path governing the behavior of the Maxwell current density.

Therefore, if the limiting factors (peak surge impedance) could be identified and incorporated in an atmospheric electrodynamic model, the electric fields along the entire streamline could be easily derived.

\section{Modeling of the Thunderstorm}

The simulated thunderstorm's electrical activity is sustained by a constant current generator that exists between the upper and lower charge centers $(6$ and $10 \mathrm{~km}$ respectively, upper center positive). Intra-cloud lightning, resulting from the accumulation of the generator charge, occurs at time intervals determined primarily by the charging current and amount of charge exchanged per flash. For the purposes of modeling, the effects of the constant current generator will be analyzed separately from that of the charge perturbation associated with intra-cloud lightning. The resulting steady state and transient solutions will then be superimposed to determine the total electrical rosponse of interest [Baginski, 1987].

Several implicit assumptions used in the modeling should be noted. The net amount of charge accumulation at either of the charge centers due to the thundercloud generator current and the neutralization of charge by lightning was assumed to be zero. This required that the time iverigy amount of charge neutrailized by lightning equaled the amoun lewcited by the thundercloud current generator. In the modeling a one ampere charging current was used. Charge accumulation at the charge centers occurred untii a breakdown field strength was obtained [pg 72, Cman, 1987!. The resulting steady state field mapping at the time breakiown was reached was used as the steady state component of the :imulated rield signatures. Specific details of the spatial and innporal distribution of the thundercloud's charge used in the modeling 
The computer model used in the study was based on an adaptation of an earlier computer code that was used to simulate lightning induced field signatures in the middle atmosphere and ionosphere [Baginski et al., 19881. The major changes necessary were to the inclusion of the thundercloud's current generator as an additional electrical source and use of a conductivity profile based on measurements. Since the earlier code simulated transient behavior that was observed at greater distances from the source and had much shorter durations, no changes were required in time step or spatial discretization used.

\section{Charging Mechanisms}

In thunderstorm research, the most difficult phenomena to explain have been the processes involved in cloud electrification. The difficulty is twofold: on the one hand, there are a large number or possible mechanisms responsible for charge separation and current generation; on the other hand, it is usually impossible to isolate such mechanisms and test each for its relative effect. Regardless of the mechanisms, what is known is that a thunderstorm is sustained by charge separation which can be approximated by net positive and negative charge centers. The height of the charge centers is somewhat affected by seasonal changes and the geographic location. Typical heights of $10 \mathrm{~km}$ for the upper charge center and $6 \mathrm{~km}$ for the lower charge center ane widely found in the literature and were selected for this research Ipp j0-5́i, Uman, 19871.

1.:sording to measurements, the amount of charge neutralized by ligntning, and the thunderstorm's current generator will vary over a considerably larger range than the charge center's heights. For insiance, kasimer $195 y$, has measured values of cloud electrification currents from less than 0.1 ampere to 10 amperes. Lightning return stroke curpents are reported to have an even larger range of values Ipp 16i-16? Iman, 19871. It was therefore necessary tc carefully consider the affects the temporal signature and magnitude of these sources would have on the simulations of interest prior to their specification in the moricil. 
Since the research presented here focused on field recovery behavior at times $\sim 100 \mathrm{~ms}$ after the cessation of the lightning current, the temporal variation of the lightning current was assumed to have little effect on the simulations of interest. In fact, after the modeling and comparative studies were completed, a simulation was done where the time variation of the charge neutralized (source of transient) by lightning was described mathematically by a simple impulse $(\delta(t))$ and resulted in field recovery curves that were identical.

Sunde's (1968) lightning current model was used in the study in the form shown below:

$$
I_{l c}(t)=I O(\exp (-a t)-\exp (-b t))
$$

where $I_{\text {ic }}(t)=$ return stroke current (Sunde's model)

$$
\begin{aligned}
a= & 10^{4} \text { seconds }-1 \\
b= & 0.5 \times 10^{6} \\
\text { IO }= & \text { proportional to amount of charge } \\
& \text { displaced during return stroke }
\end{aligned}
$$

Sunde's model consists of two exponential terms and is relatively simple compared to some of the more recent models [pp 330-334 Uman, 1987]. For the time frames of interest in this study $(t>100 \mathrm{msec}$ ), it included the necessary temporal information required for late time transient electromagnetic analysis.

The charge neutralized by the lightning current (charge perturbation) was described in terms of the temporal behavior of this current as follows $\left(\mathrm{I}_{\mathrm{iC}}=\mathrm{dQ}_{\mathrm{f}} / \mathrm{dt}\right)$ :

$$
Q_{f}(t)=\int_{0}^{t} I_{i c}(\tau) d \tau
$$

$$
\text { where } \begin{aligned}
I_{i c}(\tau)= & \text { lightning current } \\
Q_{f}(t)= & \text { total amount of charge neutralized } \\
& \text { by lightning }
\end{aligned}
$$


The neutralization of charge by lightning occurs over the entire charge center and the possible effects of its spatial distribution must be considered in any thunderstorm electrical modeling. Its spatial profile will obviously strongly influence field behavior within the cloud, but at distances much larger than the charge centers exteni (maximum feature size), its distribution will have little effect on the transient signatures [Baum, 1980]. Since the electric fields of interest were at least $20 \mathrm{~km}$ from the charge center (charge center's maximum feature size $\sim 2 \mathrm{~km}$ ) there was a certain degree of freedom in the specification of the distribution of neutralized charge. Also, since there is virtually no published data describing the charge neutralization within the cloud the selection was even more arbiirary. A modified Gaussian spatial profile was chosen for the modeling and has been usid in the modeling of many man-made and naturally occurring forced charge events [Baum, 1980].

The Gaussian profile used to describe the charge neutralized by lighting was also assumed for the charging caused by the thundercloud generator current. Here too, many possible profiles could have been used and for simplicity the Gaussian profile was selected. The mathematical description used in the modeling is shown below:

$$
\begin{gathered}
\pm \partial \rho_{f}(r, z, t) / \partial t= \pm\left(\partial Q_{f}(t) / \partial t \pm \alpha\right)(f(r, z)) \\
f(r, z)=\left(\exp \left(-R^{2} /(2 \lambda)\right)\right) /(2 \pi \lambda)^{1.5} \\
\text { where } \lambda=\text { variance }\left(\lambda=4000 \mathrm{~m}^{2} \text { for simulations }\right) \\
\qquad R^{2}=r^{2}+\left(z-z^{\prime}\right)^{2} \\
z^{\prime}=\text { altitude of charge center }(6 \text { or } 10 \mathrm{~km}) \\
\alpha=\text { term arising from steady state charging current }
\end{gathered}
$$

$( \pm 1 \mathrm{~A}$ used in the modeling)

A wlindrical coordinate system was used in the model with symmetry assumed about the vertical ( $z$ ) axis (Figure 1). The effect of the magnitudes of the charge sources on the solutions will be discussed in a later section. 
It is well known that the electric field recovery following lightning is a result of the neutralization of charge by the return stroke [pp 171-172 Uman, 1987]. The field's behavior is dictated primarily by the atmosphere's conductivity, the amount of charge neutralized by lightning and the magnitude of the thunderstorm's current generator.

\section{Geometry of the Region}

Before describing the geometry, consider again the phenomenon of interest: charge sources, located at altitudes no greater than approximately $10 \mathrm{~km}$, induce electric fields throughout the atmosphere, but only those fields induced within the middle atmosphere were of interest in this study. Therefore, the model constructed must meet two criteria:

1) If not obviously constrained, the geometrical limits of the model will approximate the entire atmosphere's electrical effect on the regions where the simulations take place.

2) The boundary conditions of the region will be electrically equivalent to those of the atmosphere.

The region selected (Figure 1) is contained within a right circular cylinder with a radius of $80 \mathrm{~km}$ and height of $110 \mathrm{~km}$. A discussion of how each of the boundaries was arrived at follows:

- Lower Plate - The earth's surface was electrically modeled as a perfect conductor. This assumption was based on the very large difference that exists between the earth's conductivity and adjacent atmosphere's conductivity. Typically, values of $10^{-3}$ to $10^{-2}$ mhos/meter are used for the earth's conductivity, while $10^{-14}$ to $10^{-13}$ mhos/meter is the usual range of the adjacent atmosphere's conductivity [pg 225, Volland, 19841. This difference of more than 11 orders of magnitude makes the earth appear (electrically) as a perfect conductor. This assumption is commonly used in practical antenna engineering for frequencies whose range would correspond to time scales of $10^{-9}$ io $10^{\circ}$ seconds [Baum, 1980l, ranges well beyond the maximum and minimum time scales of this research (minimum time step used in the research is $5 \times$ $10^{-.5}$ seconds with a total duration 20 seconds.). 
- Upper Plate - The selection of $110 \mathrm{~km}$ for the height of the upper boundary was a necessary consequence of the atmospheric conductivity structure being complicated by the Hall and Pederson components above an altitude of approximately $70 \mathrm{~km}$ [Volland, 1984]. The tensor conductivity components result when the mean free palh and velocities of the charged carriers are sufficient to allow their trajectories to be altered by the effect of the earth's magnetic field [Stratton, 1941]. The finite element routine employed in the solution is not capable of modeling tensor conductivities, therefore an altitude limit must be set in the vicinity of $70 \mathrm{~km}$.

The $110-\mathrm{km}$ altitude was selected based on the following considerations:

1) The relative magnitude of the two tensor conductivity components is approximately proportional to the additional distance in altitude (beyond $70 \mathrm{~km}$ ) considered. The maximum vaiue of either of these components with respect to the parailel conductivity's magnitude (for the range of altitudes considered) is less than 20 percent [Volland, 1984].

2) The middle and upper atmospheric electric fields resulting from lightning (with the exception of the $\sim 10^{-4}$ propagating component) are approximately verticaily oriented [Volland, 1984], i.e., the horizontal component is negligible.

3) The off-diagonal tensor components of the conductivity will only interact with electric fields that are not aligned with the earth's magnetic field [Volland, 1984]. Since the earth's magnetic field with the exception of the equatorial regions, is primarily vertically aligned [Chalmers, 1967], the influence of both the Pederson and Hall components on the lightning induced vertical electric fields will be, to first orier, negligiole.

An obvious concern is the influence this $110-\mathrm{km}$ altitude limit may have on the simulations. To investigate the maximum possible error iassuming an electrically passive ionosphere) that this would introduce in the solutions, two sets of simulations were done with the 110-km upper plate electrically described by : 1) The vertical electric field and charg' density are set to a value of zero. 2) The divergence of the electric ficld is set to the value of the charge ciensity divided by the 
and charge density are set to a value of zero. 2) The divergence of the electric field is set to the value of the charge density divided by the permittivity of free space $(\nabla \cdot E=\rho / \varepsilon)$. When the results were compared, little, if any, difference could be seen for the time frames of interest in any of the cases (all cases were subject to this test).

The probable reason for this behavior is that, in general, for lightning-induced transients, the electrical properties of the atmosphere below the point of observation of the field, rather than above, govern the transient response [Hale and Baginski, 1987]. This may be explained by simply considering the fact the conductivity rapidly increases with altitude (i.e., resistivity decreasing), and therefore its influence (restrictive effect) on total global charge movement decreases. Hence, it seems reasonable to assume the middle atmosphere's simulated response to low-altitude charge perturbations is governed by the adjacent and lower altitude conductivity values.

The conductivity used in the previously referenced high altitude simulations [Baginski et al. 1988] was slightly modified to depict the observed conductivity [Blakeslee et al., 1988] and used for the study in the form shown below (Figure 2):

$$
\begin{aligned}
& \sigma(z)=1.3875 \times 10^{-14} /(F 1+F 2+F 3+F 4) \\
& \text { where } \quad \begin{aligned}
F 1 & =2.94 /\left(\exp \left(4.5 \times 10^{-3} \times z\right)\right) \\
F 2 & =1.39 /\left(\exp \left(0.375 \times 10^{-3} \times z\right)\right) \\
F 3 & =0.369 /\left(\exp \left(0.12987 \times 10^{-3} \times z\right)\right) \\
F 4 & =9.0 /\left(\exp \left(2.777 \times 10^{-3} \times z\right)\right)
\end{aligned}
\end{aligned}
$$

- Outer Cylincirical surface - The lateral boundary had no distance constraint and could have been extended indefinitely. However, there exists a trade-off between accuracy and resolution: the smaller the model dimensions, the greater the accuracy in solving the differential equations. Therefore, the errors resulting from the adoption of finite boundaries for the model must be weighed against those resulting from degrading the numerical resolution of the simulation by involving too large a volume. 
The simulations were found to be insensitive to increases in the radial limit beyond $70 \mathrm{~km}$ for all cases. No visible difference could be detected in the responses using either $70-\mathrm{km}$ or $80-\mathrm{km}$ radial boundaries when plotted together. Therefore, selecting a $80-\mathrm{km}$ radial limit is a measure taken to provide additional confidence in the simulations.

- Axis of Symmetry (z-axis) - Since $r=0$ defines an axis of symmetry and since there are no discontinuities in the charye distribution, the derivative of the vertical electric field $\left(\partial E_{z} / \partial r\right)$ with respect to radial distance reduces to zero on the axis.

The differential forms of the four boundaries are summarized as follows:

1) At $\mathrm{ARC}=+1(z$-axis $), \quad \partial \mathrm{E}_{z} / \partial \mathrm{r}=0$

2) At $A R C=+2$ (lower boundary), $E_{r}=0$, equal potentia! surface

3) At ARC $=-3$ (outer radial boundary), $\rho=E_{z}=0$

4) At ARC $=+4$ (upper boundary) $\nabla \cdot E=\rho / \varepsilon_{0}$

\section{Maxwell's Equations}

From Maxwell's Equations a single equation is developed in which the electric field is dependent on the source charge and current densities as follows [Holzworth and Chiu, 1982):

$$
\begin{gathered}
\nabla \times \nabla \times E=-\mu_{0}\left(\sigma \partial \mathrm{E} / \partial \mathrm{t}+\partial \mathrm{J}_{\mathrm{s}} / \partial \mathrm{t}+\varepsilon_{0} \partial^{2} \mathrm{E} / \partial^{2} \mathrm{t}\right) \\
\nabla \rho / \varepsilon_{0}=\nabla^{2} E-\mu_{0}\left(\sigma \partial E / \partial \mathrm{t}+\partial \mathrm{J}_{\mathrm{S}} / \partial \mathrm{t}+\varepsilon_{0} \partial^{2} \mathrm{E} / \partial^{2} \mathrm{t}\right)
\end{gathered}
$$

where $J_{s}=$ source current density associated with high intensity lightning current, was neglected in the simulations $\rho=$ charge density

The continuity equation. is derived by taking the divergence of the Maxwell current density:

$$
0=\nabla \cdot \nabla \times H=\nabla \cdot\left(\sigma E+\varepsilon_{0} \partial E / \partial t+J_{s}\right)
$$




$$
0=\rho \sigma / \varepsilon_{0}+\nabla \sigma \cdot E+\partial \rho / \partial t+G_{S}
$$

$$
\text { where } \begin{aligned}
\nabla \cdot J_{S}= & G_{S}=\text { source of charge perturbation } \\
& \text { (deposition of charge by the lightning current and } \\
& \text { thunderstorm current generator) }
\end{aligned}
$$

The above equations were derived from Maxwell's equations and were used to describe the electrodynamic response of the atmosphere to the thunderstorm. It is important to note the primary difference in the forcing function $G_{S}$ (charge related) used here relative to forcing functions used for most spheric analysis (typically current-related forcing functions). A current related transient (e.g., current loop) is allowable in a charge neutral region, but charge movement through space does not necessarily imply a zero volume charge density. Furthermore, a charge perturbation may be considered separately from the currents which cause it (charge perturbation implies charge generation at a point in space). In the latter case charge motion is required (conservation of charge), but the relative contribution of each (charge versus current) mechanism to the resulting total electric field may be analyzed separately. Initially (prior to the first iterative time step of the transient analysis), no charge is displaced and the transient electric rield everywhere is assumed zero.

Equations 6 and 8 used in the modeling are linear partial differential equations. This fact greatly reduced the degree of complexity in the thunderstorm electrical modeling. Linearity allows the use of superposition to construct the total solution desired based on each source's indiviaual response. The amplitude of the solution is also direcitly proportional to the source's magnitude. This was especially important in the comparative analysis when the specific thunderstorm's current generator and amount of charge neutralized by lightning were derived. 


\section{SIMULATIONS}

The vertical component of the simulated Maxwell current density signatures are shown in Figure 3 for altitudes of 46,53 , and $60 \mathrm{~km}$ at a radial distance of $15 \mathrm{~km}$ from the vertical axis of symmetry. The waveforms show only slight differences in both magnitude and temporal character. Vertical electric field signatures at the same positions show relatively large variations in both magnitude and temporal character (Figure 4).

Figure 5 and 5 identify the normalized Maxwell current density and electric field for altitudes of $40-60 \mathrm{~km}$ and radial distances of $15-30$ $\mathrm{km}$ at $\mathrm{t}=200$ milliseconds. The interesting feature is that the Maxwell current density and electric field are not aligned. This observation is of importance to experimentalists who typically infer field characteristics from a single measured quantity ( $E$ and $\mathrm{J}_{\mathrm{m}}$ ).

The simulated vertical electric field signatures (Figure 4) are based on conductivity measurements obtained concurrently with with transient electric field data. There are two traits common to both measurements and simulations that are not explainable in terms of simple relaxation time solutions: the significant time delay prior to the onset of the maximum field strength, and the relatively long duration of the transient. This fact indicates that the use of relaxation time solutions would not be valid for the study conducted.

\section{CONCLUSIONS}

itie indor contribution of the study is to emphasis the generai
importance modeling has in identifying the allcwable atmospheric
transient, behavior. This reduces the researchers need to rely on
assumptions that are in many cases not rigorously justifiable. It is
probable that the next major advances in atmospheric electricity will
involve numerical simulation predictions of otherwise unexpected
behavior.
Lightning researchers may benefit from the relative spatial and


temporal invariance of the Maxwell current density with respect to the corresponding electric field signature. It is likely that this type of characterization would provide an alternative fundamental description of the lightning event that would greatly reduce the complexity of much current thunderstorm electrical research. 


\section{REFERENCES}

Anderson, F.J. and G.D. Freier, "Interaction of the Thunderstorm with a Conducting Atmosphere," J. Geophys. Res., 74, 5390, 1969.

Baginski, M.E., "Finite element simulation of the atmosphere's electromagnetic response to charge perturbations associated with lightning," Ph.D. thesis, Pennsylvania State University, 1987.

Bąeinski, M.E., L.C. Hale, and J.J. Olivero, "Lightning-Related Fields in the Ionosphere," Geophys. Res. Let., August, 1988

Baum, C.E., "Simulation of Electromagnetic Aspects of Lightning," Proc. of Lightning Tech., NASA Conf. Pub. 2128, April 1980.

Blakeslee, R.J., H.J., Christian, and B. Vonnegut, "Electrical Measurements Over Active Thunderstorms," submitted to J. Geophys. Res., September 1988.

Browning, G. L., I. Tzur, and R.G. Roble, "A Global Time-dependent Model of Thunderstorm Electricity. Part I: Mathematical Properties of the Physical and Numerical Models," JAS., 49, 2166,1987

Chalmers, J.A., Atmospheric Electricity, 2nd ed., Pergamon, New York, 1967.

Gish, O.H., and R.G. Wait, "Thunderstorms and the earth's general electrification," J. Geophys. Res., 55, 473, 1950.

Golde, R.H., Lightning, Volume I., Academic Press, 1977. 
Greifinger, C., and P. Greifinger, "Transient ULF

Electric and Magnetic Fields Following a Lightning

Discharge," J. Geophys. Res., 81, 2237, 1976.

Hale, L.C., and M.E. Baginski, "Current to the ionosphere following a lightning stroke," Nature, 329, 814, 1987

Holzer, R.E. and D.S. Saxon, "Distribution of electrical conduction currents in the vicinity of Thunderstorms," J. Geophys. Res., 57, 207, 1952.

Holzworth, R.H. and Y.T. Chiu, "Spherics in the Stratosphere," in CRC Handbook of Atmospherics, volume one, H. Volland, CRC Press, Boca Raton, Florida, 65, 1982.

Illingworth, A.J., "Electric Field Recovery After Lightning as the Response of a Conducting Atmosphere to a Field Change," Quart. J. Roy. Meteorol. Soc., $98,604,1972$.

Israel, H., Atmospheric Electricity, Israel Program for Scientific Translations, vol. 2, Jerusalem, 1973.

Kasemir, H.W., "The Thunderstorm as a Generator in the Global Electric Circuit (in German)," Z. Geophys., 25, 33, 1959.

Krider, E.P., and J.A. Musser, "Maxwell currents under a thunderstorm," J. Geophys. Res., '11, 171-176, 1982.

Nisbet, J.S., "A dynamic model of thundercloud electric fields," J. Atmos Sci., 40, 2855, 1983. 
Park, C.G., and M. Dejnakarintra, "Penetration of Thundercloud

Electric Fields into the Ionosphere and Magnetosphere

1. Middle and Subauroral Latitudes," J. Geophys.

Res., 78, 6623-6633, 1973.

Stratton, J.A., Electromagnetic Theory, McGraw-Hill, New York, 1941.

Stremler, F.G., Introduction to Communication Systems, Addison-Wesley, California, 1977.

Sunde, E.D., Earth Conduction Effects in Transmission Systems, Dover, New York, 1968.

Tamura, Y., "An Anaiysis of the Electric Field After Lightning Discharges," Geophys. Res. Pap., 42, 190-200, 1955.

Tzur, I. and R.G. Roble, "The Interaction of a Dipolar Thunderstorm with its Global Electric Environment," J. Geophys. Res., 90, 5989, 1985.

Uman, M.A., Lightning, McGraw-Hill, New York, 1987.

Volland, H., Atmospheric Electrodynamics, Chemistry in Space, vol. II, Springer-Verlag, Berlin, Germany, 1984.

Willett, J.C., "Naxwell Currents and the Thunderstorm Generator," presented at 1984 AGU Fall Meeting, San Francisco, California

Wilson, C.T.R., "Some Thundercloud Problems,"

J. Franklin Inst., 208, 1916.

ORIOUAR PRTE is of POSh Gan? 


\section{ELECTRICAL MODEL OF ATMOSPHERE}

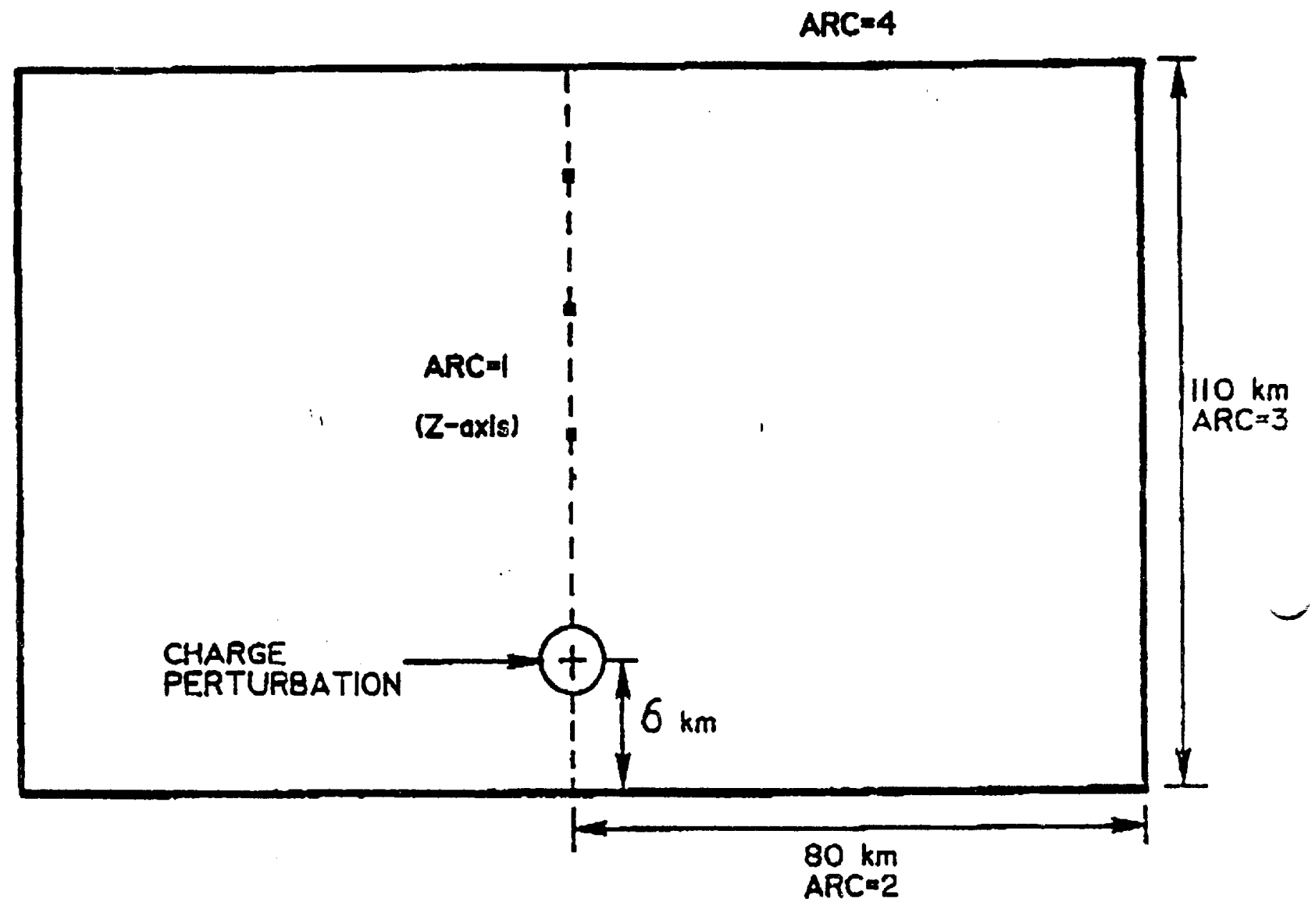

Figure 1 Electrical Model of the Atmosphere. 


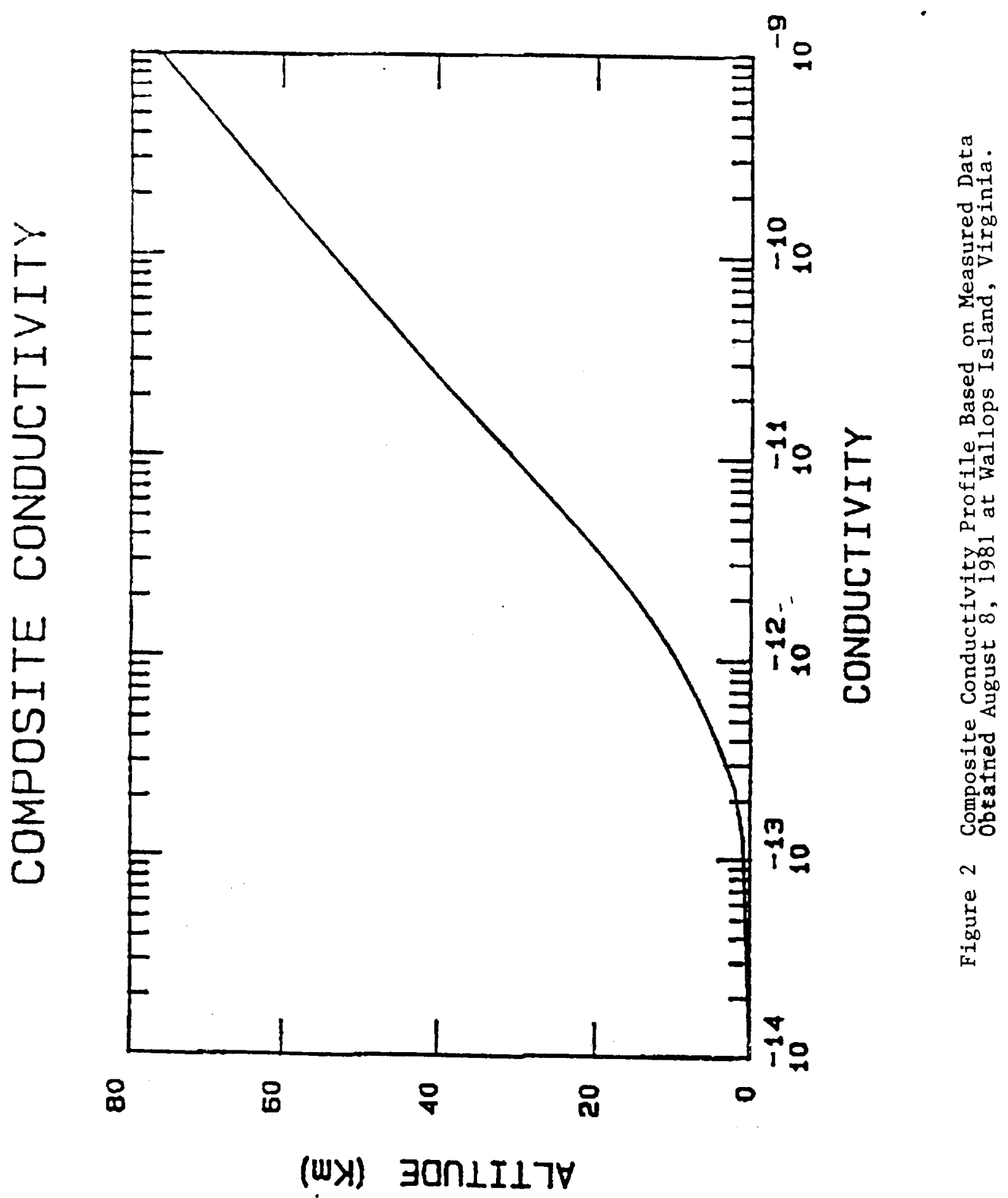




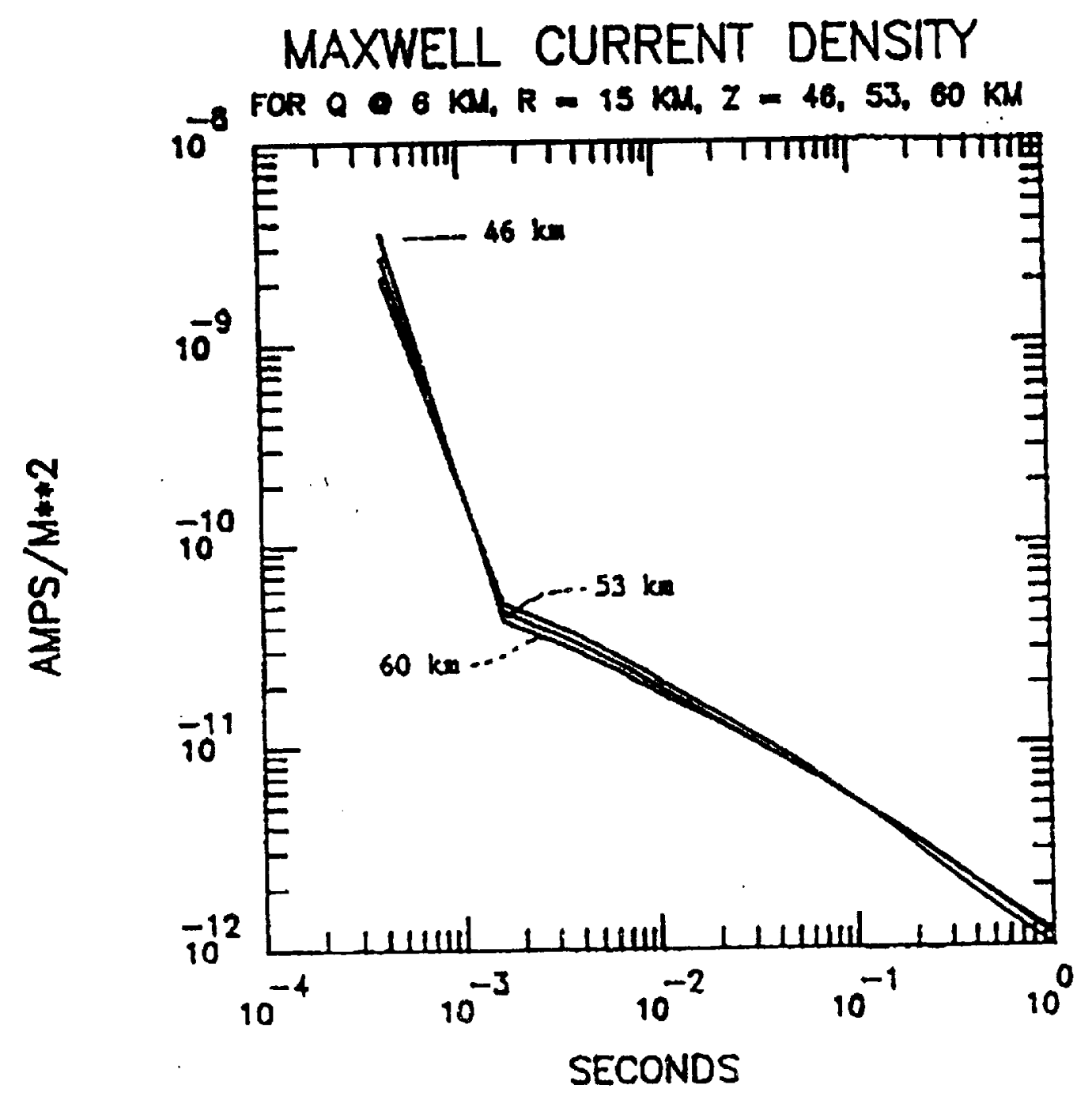

Figure 3 Maxwell Current Density Simulations at a Radial Distance of $15 \mathrm{~km}$ for Altitudes of 46. 53, and $60 \mathrm{~km}$. 


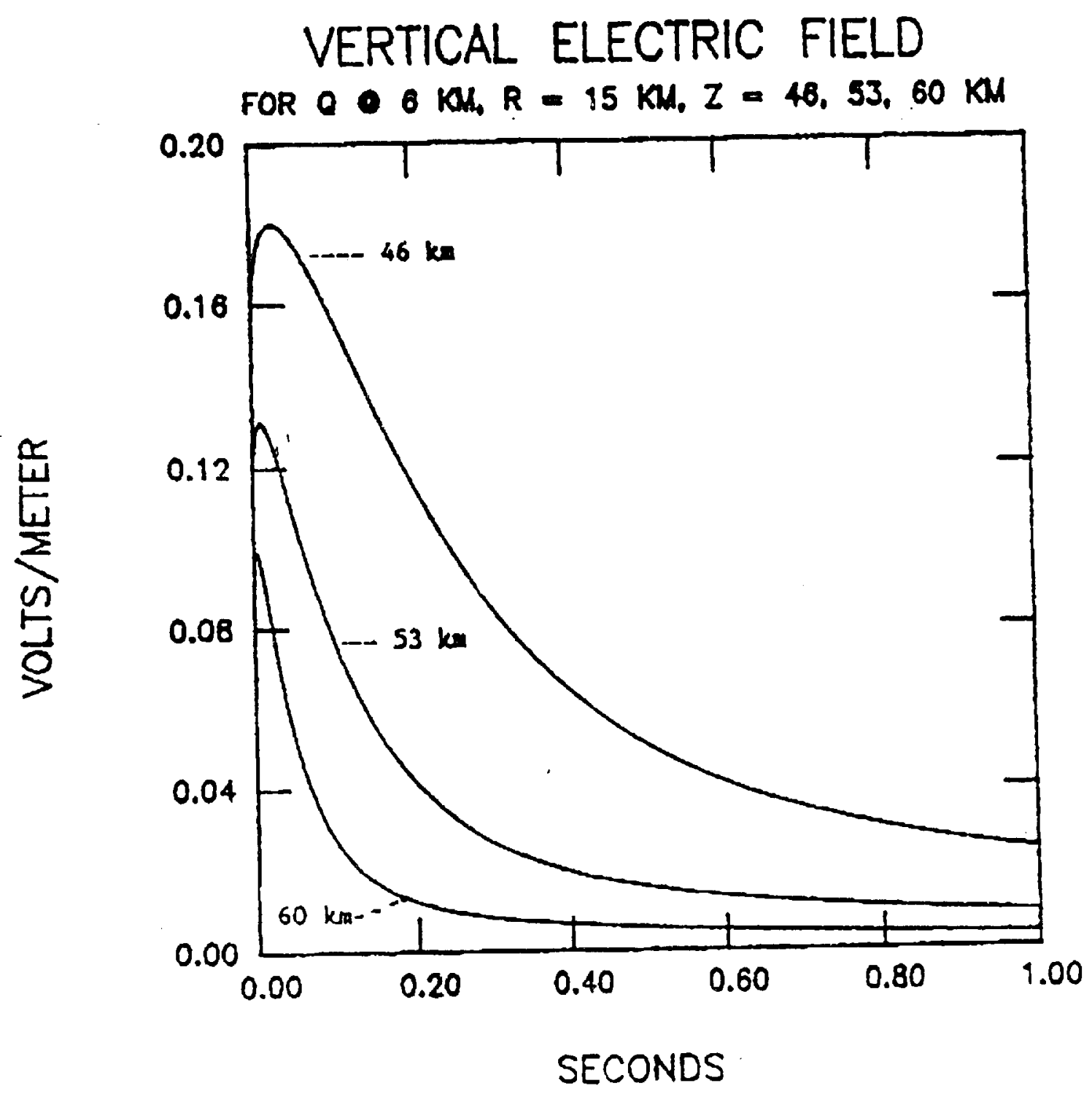
Figure 4 Electric Fiold Simulations at a. Radial Distance
of $15 \mathrm{~km}$ for Altitudes of 46,53 , and $60 \mathrm{~km}$. 


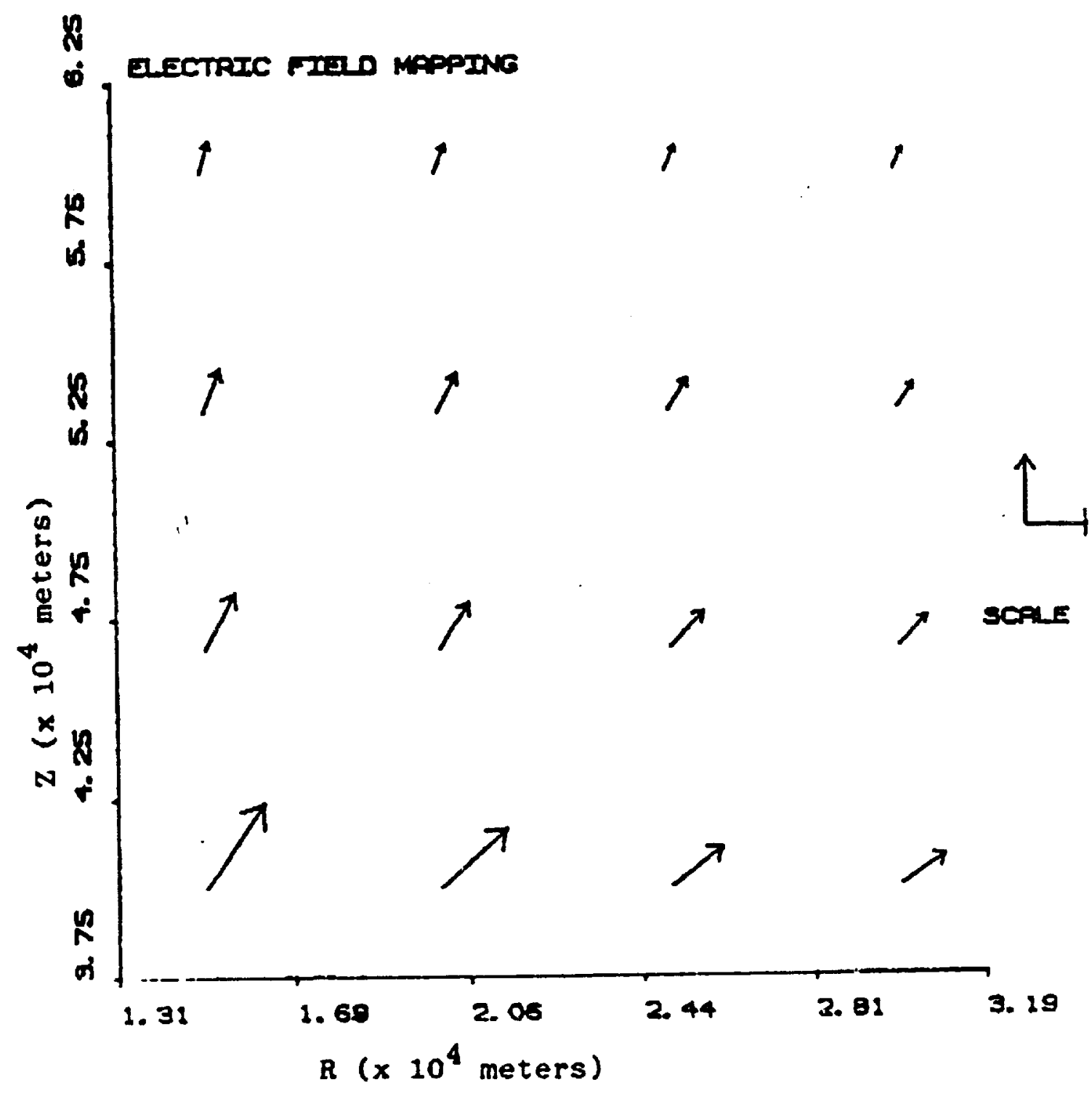

Figure 5 Normalized Simulated Electric Field Mapping for $t=200 \mathrm{mi} l 1$ iseconds. 


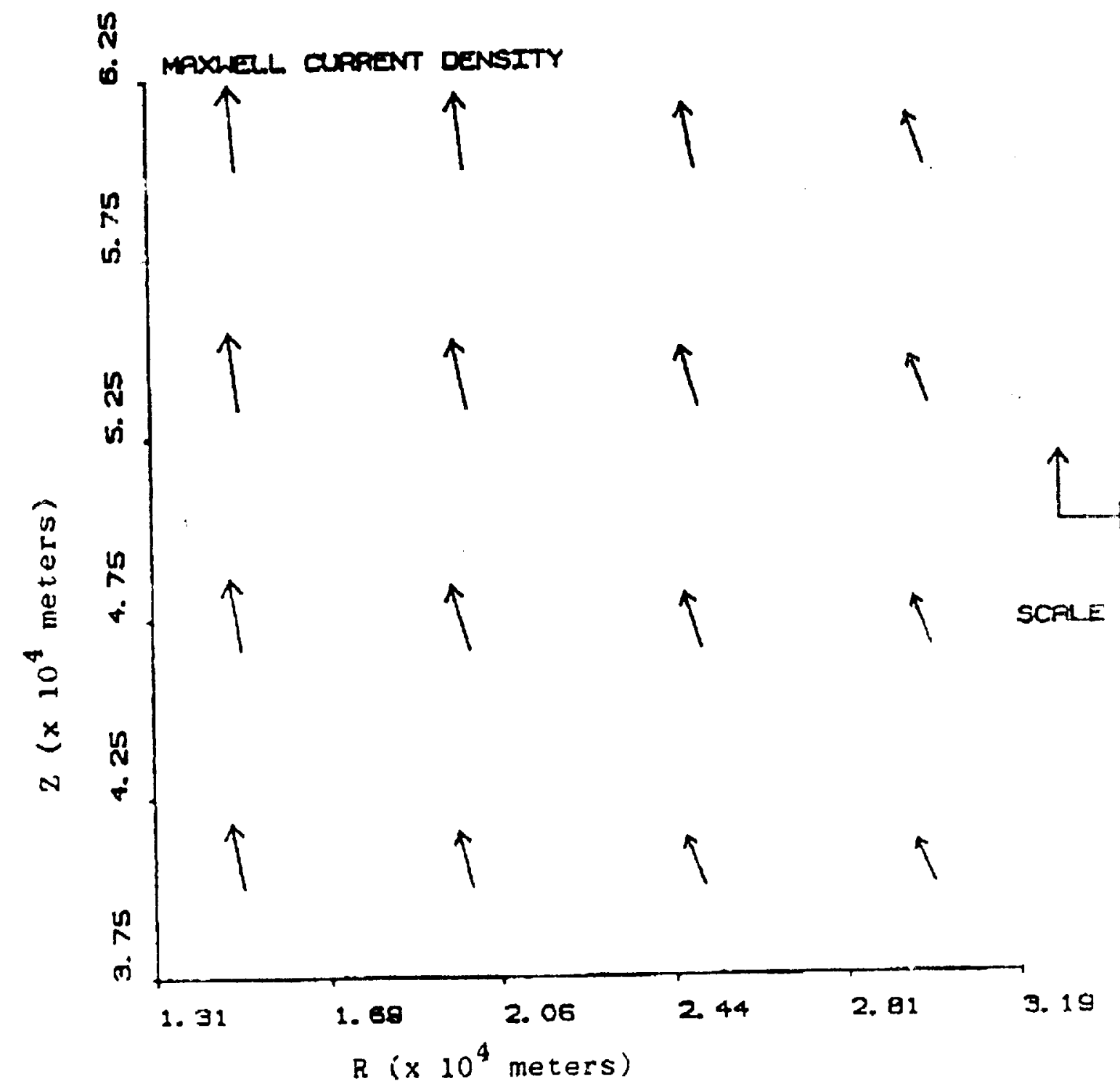

Eigure 6 Normalized Simulated Maxwell Current Density Mapping for $t=200 \mathrm{milliseconds}$. 
